\title{
Enfermedad de Chagas en Nicaragua: actualidades
}

\section{Carlos N. Talavera-López y Jorge A. Huete-Pérez}

Programa de Enfermedades Infecciosas, Centro de Biología Molecular, Universidad Centroamericana, Rotonda Rubén Darío, 500 mts al oeste, Apto. Postal 69. Managua, Nicaragua. Tel: 278-3923 Ext. 1189. Correo electrónico: ce.noguera@gmail.com, huete@ns.uca. $\underline{\text { edu.ni }}$

LA ENFERMEDAD DE CHAGAS ES UN PROBLEMA DE SALUD pública en toda Latinoamérica; alrededor de 20 millones de personas están infectadas y 200 millones están en riesgo de contraer la enfermedad. En 2006, la prevalencia en Centroamérica era del 7\%. Actualmente no existe vacuna contra el protozoo y el tratamiento disponible resulta, aparte de poco efectivo, muy tóxico para el paciente. Los programas de control de vectores han ayudado a reducir los índices de infestación en Latinoamérica, pero aún falta mucho por hacer. En Nicaragua, la enfermedad de Chagas está subvalorada y los trabajos publicados son muy pocos. Es necesario investigar sobre esta enfermedad en nuestro país con otro enfoque, uno que no subvalore la enfermedad y ayude a desarrollar métodos diagnósticos y posibles tratamientos. Este artículo recopila información sobre los trabajos realizados por los grupos más importantes de investigación en Chagas de Nicaragua en cuanto a epidemiología, control vectorial, diagnóstico y caracterización molecular.

Palabras clave: enfermedad de Chagas / Nicaragua / salud pública

\section{Introducción}

La enfermedad de Chagas afecta a más de 20 millones de personas en Latinoamérica y aproximadamente 200 millones se encuentran en riesgo de infectarse (WHO, 2005). No existen datos actualizados sobre la prevalencia de Trypanosoma cruzi en Nicaragua. Los datos globales publicados más recientes son de 1992; demuestran apenas el 0.8 $\%$ de prevalencia global. Se han implementado varios programas para controlar esta enfermedad en el país tales como: control vectorial, fumigación casa por casa en las zonas endémicas, estudios seroepidemiológicos, evaluación de métodos diagnósticos, entre otros. Sin embargo, no se cuenta con datos recientes que den una idea precisa de la situación actual del mal de Chagas en Nicaragua. Incluso, en el Plan Nacional de Desarrollo, en el sector salud, la enfermedad de Chagas aparece como una enfermedad de poco interés por su baja incidencia. Este artículo consiste en una revisión bibliográfica de las publicaciones existentes y los proyectos ejecutados, o en ejecución, con el fin de actualizar el conocimiento sobre la situación de la enfermedad de Chagas en Nicaragua. 
Cuadro 1. Información general sobre la enfermedad de Chagas

El Trypanosoma cruzi tiene un ciclo de vida complejo pues comprende múltiples estadios morfológicos y dos fases evolutivas: una en el vector y otra en el mamífero.

Una vez dentro del humano, los trypomastigotes tienen la capacidad de infectar cualquier célula nucleada y muestran particular afinidad hacia el tejido muscular (cardíaco o liso). El parásito utiliza una enzima que también poseen los mamíferos, la trans-sialidasa, que se encarga de transferir un grupo sialil de la membrana celular del mamífero a la membrana de T. cruzi con el fin de que éste sea introducido a la célula por un proceso similar a la fagocitosis mediado por actina (Tan \& Andrews, 2002).

La unión del parásito con la membrana de la célula activa la agrupación de lisozomas en este punto, los que finalmente internalizan a T. cruzi. Al acidificarse el medio de la vacuola lisosomal, T. cruzi libera una proteína que perfora la membrana (porina), permitiéndole infectar la célula y pasar al siguiente estadio conocido como amastigote, y luego de 9 ciclos, destruir la célula y pasar al torrente sanguíneo en forma de tripomastigote circulante (Tyler \& Engman, 2001).

\section{Prevalencia}

Recientemente se encontró que la prevalencia de la enfermedad de Chagas en Centroamérica se aproxima al 7\% (Ponce et al. 2005). Ésta fue descrita por primera vez en Nicaragua en el año 1971. El último estudio a nivel nacional fue realizado por el MINSA en 1992 y mostró una prevalencia global del 0.8\% (MINSA, 2005), un valor muy bajo si se compara con los otros países de Latinoamérica. Allí se reflejó también que los departamentos más afectados eran Somoto (5.9\%), Ocotal (5.2\%) y Masaya (2.4\%).

Por otra parte, un estudio llevado a cabo en 1995 en comunidades rurales de León, Masaya y Madriz, aplicando Inmunoflourescencia Indirecta (IFI) reveló prevalencias de 3.2 \%, 4.3 \% y $13.1 \%$ respectivamente (Rivera, et al. 1995).

Otros estudios han demostrado que existe una alta prevalencia en otras comunidades rurales del país, encontrándose en cinco comunidades del Pacífico valores de hasta 21.2\%, variando en comunidades de 11.5 - 47.0 \% de la población total de dichas comunidades (Morales, 2000). Actualmente no se cuenta con datos publicados sobre la prevalencia global de la enfermedad en Nicaragua.

\section{Situación de los vectores de transmisión}

Morales (2000) menciona en sus estudios que la principal especie vectora de la enfermedad de Chagas en el país es Triatoma dimidiata (Hemíptera, Triatomiinae, Reduvidae) y en segundo lugar Rhodnius prolixus. La presencia de ambos insectos se ha confirmado 
desde el sur de México hasta Perú, Colombia y Venezuela (Acevedo et al. 2000). Otras especies vectoras en Nicaragua son: Rhodnius palescens y Triatoma infestans. Se debe de tomar en cuenta que algunos vectores son más exitosos que otros, pues se adaptan mejor al ambiente doméstico (Coura, 2007). Sin embargo, no siempre la presencia del vector está acompañada de infección.

Un estudio realizado en 1996 en las comunidades endémicas de Santa Rosa, Quebrada Honda y Poneloya, demostró que la principal especie vectora es Triatoma dimidiata y también demostró que las condiciones habitacionales no estaban necesariamente ligadas a la presencia del vector en la vivienda (Palma-Guzmán et al. 1996). Esto se debe a que muchas casas en las zonas rurales de Nicaragua presentan sitios de almacenamiento en la parte exterior, donde las especies vectoras pueden refugiarse durante el día y salir por la noche. A este tipo de sitios se les conoce en la jerga epidemiológica como ecotopes.

En 1998 se realizó una encuesta vectorial por parte del MINSA en el 5\% de las viviendas del SILAIS (Sistema Local de Atención Integral en Salud) de todo el país, mostrando un índice de infestación del 13\% y de éste, el 12\% estaba infectado por Trypanosoma cruzi (MINSA, 2005). Diez años después, se desconocen los índices de infestación globales y se sabe poco de los índices de infestación actuales en las áreas endémicas.

Se han implementado planes de control vectorial como el aplicado por el MINSA en el SILAIS de Madriz en 1999, que tuvo por objeto erradicar la presencia de Rhodnius prolixus del territorio nacional. El plan no erradicó totalmente al vector, pero sí redujo su presencia en gran medida (MINSA, 2005). En el caso de Triatoma dimidiata, la erradicación no es posible puesto que el vector tiene fuerte afinidad por los ecotopes selváticos, muy comunes en las comunidades rurales del país.

\section{Programas de control vectorial}

En el año 2002 la organización Médicos Sin Fronteras-Bélgica (MSF-B) inició un proyecto a solicitud del Programa Nacional de Control y Prevención de la enfermedad de Chagas destinado al diagnóstico y tratamiento de la enfermedad de Chagas. Este plan incluía el control del vector por fumigación y eliminación de las viviendas (MSF, 2005). Como resultado se obtuvo una disminución en los índices de infestación para las localidades de Totogalpa y Cuje en Nueva Segovia y Esquipulas en Matagalpa. Además, se implementó un manual de manejo de la enfermedad de Chagas editado por el MINSA.

Existe otro programa centroamericano patrocinado por la Agencia de Cooperación Internacional Japonesa (JICA) que tiene como objetivo la eliminación de Rodnius prolixus de los países de Guatemala, Honduras y El Salvador; pero que no incluye a Nicaragua. En este proyecto se involucraron los ministerios de salud de cada país junto a ONG y voluntarios japoneses en una gran campaña de información para la población sobre el chinche y los métodos de eliminación. El proyecto benefició a casi 2 millones de personas (JICA, 2003).

Se han probado varios métodos para evaluar su efectividad y su adecuación al presupuesto nacional de salud. Un estudio muestra que la fumigación de casas donde se ha confirmado 
la presencia del vector es la forma más efectiva en cuanto a costos y resultados de control de vectores en las comunidades rurales (Acevedo et al. 2000). Un factor importante en los programas de control vectorial es el conocimiento que tiene la población en general sobre el insecto. Muchos estudios muestran que los habitantes de zonas endémicas pueden reconocer las fotografías de los vectores e incluso saben reconocer las diferencias entre las dos especies presentes en el país.

\section{Métodos diagnósticos para la enfermedad de Chagas en Nicaragua}

\section{Serodiagnóstico}

El diagnóstico de la enfermedad de Chagas presenta varias dificultades. El diagnóstico diferencial incluye dengue, malaria, leishmaniasis visceral, entre otras. Además, la fase aguda no siempre se presenta de forma sintomática; la mayoría pasa desapercibida por la población y por el personal de salud (ver Cuadro 2). Otra dificultad es la alta variabilidad antigénica de T. cruzi, que muestra reacciones cruzadas en los ensayos serológicos con Leishmania spp (leishmaniasis) y Plasmodium spp (malaria).

Para el diagnóstico correcto de la enfermedad de Chagas se requiere de métodos clínicos, serológicos y moleculares muy específicos. Se conoce que el Centro Nacional de Diagnóstico y Referencia (CNDR) ha preparado un kit de ELISA (Enzyme-Linked Immunosorbent Assay) para el diagnóstico de anticuerpos contra T. cruzi en bancos de sangre del sistema de salud nacional. El CNDR reporta que este kit muestra una sensibilidad del 100\% y especificidad del 98.4\%, y que ha sido evaluado por laboratorios de Guatemala y Honduras (IDEASS, 2004).

El MINSA/CNDR Chagas kit utiliza antígenos de parásitos recuperados de la sangre de pacientes en fase aguda, cultivados y luego purificados. No obstante, se ha señalado que el cultivo provoca selección clonal, permitiendo que otros clones distintos a los originales se desarrollen. No se ha publicado el estudio de caracterización de las cepas de estos parásitos. Existe otro kit, el ChagaTest ${ }^{\circledR}$ (Wiener, Argentina), que utiliza antígenos obtenidos por tecnología de $\mathrm{ADN}$ recombinante que ha mostrado excelentes resultados. Pero se ha señalado que la aplicación de un ELISA tradicional y un ELISA recombinante juntos mejora la sensibilidad y especificidad del diagnóstico serológico (Gadelha et al. 2003).

Por otra parte, el CNDR participó en la validación de un método inmunocromatográfico para el diagnóstico rápido de la enfermedad de Chagas, en la cual participaron los centros de referencia de toda Centroamérica. Según este estudio, el test inmunocromatográfico tiene una especificidad y sensibilidad similar a la del ELISA y es una alternativa para el diagnóstico en una emergencia transfusional (Ponce et al. 2005). 
Cuadro 2. Respuesta inmune

Cuando el Trypanosoma cruzi invade al ser humano, se enfrenta a una respuesta inmune innata muy potente. El sistema inmune puede operar contra T. cruzi de tres formas (Tarleton, 2007):

- Detección y destrucción directa por macrófagos $(M \varnothing)$ y células dendríticas (CD).

- Activación de MØ y CD como potentes presentadores de antígenos que mejoren la respuesta inmune.

- Sensibilización de miocitos, que son el principal objetivo de T. cruzi.

Las zonas que son más inmunógenas en la superficie de la membrana de T. cruzi son las que contienen gran cantidad de glicofosfatidilinositol (GPI) de la familia de las mucinas que activan los Toll-Like Receptor-2 (TLR2) para producir interleucina-12 (IL-12), oxido nítrico y factor de necrosis tumoral (TNF) por los macrófagos (Campos et al. 2001). Sin embargo, T. cruzi posee mecanismos que eluden la respuesta inmune celular, como es la variación antigénica y la mimetización con antígenos propios del humano como el ácido siálico de las membranas celulares. Se ha demostrado que el daño producido a los órganos como el corazón en la fase crónica se debe a reacciones inflamatorias multifocales progresivas (Morris et al. 1990).

La infección por T. cruzi tiene tres fases: aguda, indeterminada y crónica (ver Cuadro 3). En la fase aguda, el paciente puede presentar sintomatología o no y rara vez muere. Las principales complicaciones de la fase crónica son los megasíndromes: megaesófago, megacolon y cardiomegalia, aunque en algunos casos puede haber infección crónica del Sistema Nervioso Central. Estas complicaciones generalmente se desarrollan en el 30\% de los casos, y de éstos, 10\% son mortales (Kirchoff, 2006).

Cuadro 3. Manifestaciones clínicas de la enfermedad de Chagas

\begin{tabular}{|c|c|c|c|}
\hline \multirow[t]{3}{*}{ Fase } & Más común & Menos común & Observaciones \\
\hline & Chagoma o Romaña & Meningoencefalitis & La principal causa de \\
\hline & Linfadenopatía local & Linfadenopatía general & muerte en la fase \\
\hline \multirow[t]{3}{*}{ Aguda } & Malestar y fiebre & Hepatoesplenomegalia & aguda se debe a \\
\hline & Anorexia & Miocarditis & insuficiencia cardíaca \\
\hline & Edema & & \\
\hline \multirow[t]{3}{*}{ Indeterminada } & Asintomática & Asintomática & \\
\hline & Arritmias & & \\
\hline & Miocardiopatías & Caquexia & Período de inicio \\
\hline \multirow[t]{4}{*}{ Crónica } & Tromboembolias & Pérdida de peso & prolongado \\
\hline & Disfagia & Estreñimiento crónico & \\
\hline & Dolor toráxico & Neumonitis por aspiración & \\
\hline & Regurgitación & & \\
\hline
\end{tabular}




\section{Tratamiento farmacológico de la enfermedad de Chagas}

En la actualidad se cuenta solamente con dos fármacos para tratar la enfermedad de Chagas: Benznidazole y Nifurtimox. En Latinoamérica sólo se cuenta con Benznidazole como tratamiento para Chagas en fase aguda, mientras que Nifurtimox es la única aprobada en los Estados Unidos (Doyle et al. 2007). Estos son compuestos nitroheterocíclicos que poseen distintos mecanismos de acción.

En el caso de Nifurtimox un grupo nitro se reduce a radicales nitroaniónicos inestables que producen metabolitos reducidos de oxígeno altamente tóxicos que Trypanosoma cruzi no puede eliminar y como resultado se vuelve más susceptible que las demás células de los vertebrados.

El Benznidazole utiliza un mecanismo conocido como stress reductivo que consiste en la modificación covalente de macromoléculas por intermediarios de nitroreducción (Urbina \& Docampo, 2003).

Ambos medicamentos poseen una actividad de alrededor del $80 \%$ en la fase aguda, sin embargo poseen poco o ningún efecto en la fase crónica. Se han identificado variaciones en la respuesta terapéutica en distintas zonas geográficas. Se cree que esto se debe a la diversidad de cepas circulantes (Andrade et al. 1992). Estos medicamentos poseen efectos muy tóxicos en los pacientes que los toman porque las vías metabólicas de los Kintoplastidae son muy similares a las de los mamíferos, lo que incrementa la deserción al régimen terapéutico que tarda más de un mes, lo que disminuye aún más la probabilidad de cura.

Es imperativo que se desarrollen nuevos fármacos que eliminen al parásito -tanto en la fase aguda como en la fase crónica- y no posean efectos secundarios tan dañinos al ser humano. Sin embargo, este objetivo se ve impedido por la naturaleza social de la enfermedad de Chagas, que se desarrolla en la población más pobre del orbe, y por la relativamente poca cantidad de enfermos que existe en comparación con otras enfermedades infecciosas.

Cuadro 4. Nuevas propuestas de tratamiento

Se han desarrollado nuevos objetivos terapéuticos que carezcan de los efectos tóxicos de los actuales agentes. Una estrategia muy prometedora es la inhibición de la mayor proteasa (enzima que digiere proteínas) de Trypanosoma cruzi conocida como cruzipain. El agente inhibior es el $N$-metil-Pip-F-hF-VSø, que según estudios recientes ha mostrado buenos resultados en ratones immunodeficientes (Doyle et al. 2007).

Otro tipo de estrategia ha sido el desarrollo de vacunas de ADN obtenidas por ingeniería genética que activen la respuesta inmune celular e inhiban el desarrollo de la infección por T. cruzi. Sin embargo, estos ensayos requieren de una mejor comprensión de la inmunología de la enfermedad de Chagas, así como la búsqueda de objetivos con alta antigenicidad (Tarleton, 2007). 


\section{Investigación actual en Nicaragua}

Existen tres grupos importantes de investigación sobre la enfermedad de Chagas en Nicaragua:

- Programa de Control y Prevención de la enfermedad de Chagas, Centro Nacional de Diagnóstico y Referencia, Ministerio de Salud (MINSA), coordinado por la Dra. Francisca Marín.

- Proyecto Chagas, Departamento de Microbiología y Parasitología, Facultad de Ciencias Médicas, Universidad Nacional Autónoma de Nicaragua UNAN-León, liderado por la M.Sc. Rosario Palma Guzmán.

- Programa de Enfermedades Infecciosas, Centro de Biología Molecular; Universidad Centroamericana (CBM-UCA), dirigido por el Dr. Jorge Huete-Pérez.

\subsection{Centro Nacional de Diagnóstico y Referencia (MINSA)}

El CNDR tiene un programa de Chagas que se encarga de la vigilancia epidemiológica nacional y del adiestramiento en métodos diagnósticos desde 1998. Sin embargo, no cuenta con un presupuesto establecido y por lo tanto no puede realizar estudios de cobertura global (MSF, 2005). A pesar de esto, el CNDR ha validado un método de diagnóstico serológico para Chagas: el MINSA/CNDR Chagas kit. Además, ha realizado estudios de carácter epidemiológico y de control vectorial. Este equipo ha trabajado en el mejoramiento del diagnóstico de la enfermedad de Chagas elaborando un algoritmo para el diagnóstico serológico de Chagas que consiste en una serie de pasos a abordar por los trabajadores de los centros de salud al momento de evaluar si un paciente es sospechoso o no de estar infectado con Trypanosoma cruzi y un manual técnico para el control de la enfermedad. Actualmente no se conoce ninguna nueva propuesta de investigación, y las aplicaciones de biología molecular están restringidas al estudio de otras enfermedades.

\subsection{Proyecto Chagas UNAN-León}

Este grupo ha sido líder en estudios de vigilancia seroepidemiológica en Nicaragua; ha elaborado gran cantidad de encuestas serológicas en varias partes del país. El proyecto Chagas UNAN-León ha coordinado varios estudios sobre tasas de infestación de casas por especies vectoras en las zonas endémicas, identificación de parásitos en las heces de los vectores, relación seropositividad y cardiopatía y diagnóstico con estudiantes de la Facultad de Ciencias Médicas de la UNAN-León. Entre 1995 y 2000 este grupo ha publicado dos artículos en revistas indexadas.

\subsection{Centro de Biología Molecular - UCA}

El CBM actualmente está llevando a cabo el proyecto "Caracterización Molecular de cepas de Trypanosoma cruzi en sangre de pacientes crónicos en dos comunidades endémicas de Nicaragua", con el fin de caracterizar las cepas y clones circulantes en la sangre de los pacientes y establecer una relación entre el tipo de clon/cepa y el cuadro clínico producido por éste. Se han elegido las comunidades de Madriz y Carazo por ser los departamentos con más alta prevalencia reportada (MINSA, 2005 y Morales, 2000). 
El proyecto consta de cinco etapas:

1. Recolección de muestras y revisión de historia clínica

2. Confirmación de diagnóstico por ELISA recombinante.

3. Detección de kDNA de T. cruzi por PCR.

4. Identificación de cepas por LSSP-PCR.

5. Identificación de cepas y clones por secuenciación del kDNA.

\section{Detección de kDNA de Trypanosoma cruzi por PCR}

La técnica de amplificación de ácido desoxirribonucléico (ADN) por reacción en cadena de la polimerasa (PCR) se aplica para detectar la presencia del parásito en varias muestras biológicas como sangre y suero. El Trypanosoma cruzi posee una sola mitocondria denominada cinetoplasto. Esta estructura posee ADN compuesto por maxi-círculos y mini-círculos agrupados entre sí en forma de malla (Shlomai, 2004). Los maxi-círculos se encuentran en menor número de copias (cerca de 35), en cambio los mini-círculos se encuentran entre 15,000 y 20,000 copias por célula. Por lo tanto, se diseñaron métodos moleculares que permitieran amplificar esta abundante fuente de ADN parasitario. Uno de los métodos consiste en amplificar la porción variable de estos mini-círculos para producir un fragmento de 330 pares de bases (pb) utilizando un par de primers o cebadores (Strum et $a l .1989)$ que es visible por electroforesis en gel de agarosa teñido con bromuro de etidio.

\section{Identificación de cepas por LSSP-PCR}

Este método consiste en la amplificación con un solo cebador de los productos de una primera PCR para detectar la presencia del parásito. Este único cebador se une a las porciones hiper-variables (HVP's) del mini-círculo de ADN del cinetoplasto produciendo un patrón de pequeños productos de amplificación específicos para cada cepa (Vago et al. 1996 y Vago et al. 2000) que pueden ser visualizados por electroforesis en gel de poliacrilamida (PAGE) teñido con nitrato de plata. Estos patrones de amplificación son comparados luego con patrones de cepas conocidas utilizando herramientas de bioinformática para lograr una identificación precisa de la cepa presente

\section{Identificación de cepas y clones por secuenciación de $k D N A$}

Con los productos de amplificación del ADN del cinetoplasto se realiza una reacción de secuenciación que consiste en determinar todas y cada una de las bases nitrogenadas que componen el fragmento de $330 \mathrm{pb}$ amplificado utilizando un secuenciador ABI Prism 3130 (Applied Biosystems). Luego, este dato se compara con secuencias de cepas y clones ya procesados y almacenados en la base de datos de GENBANK mediante herramientas de bioinformática para determinar las variaciones más mínimas entre ambas secuencias.

Con la obtención de estos datos se planea identificar las cepas y los clones circulantes entre los pacientes infectados en Nicaragua. Este es un estudio sin precedentes que permitiría abordar el aspecto diagnóstico con técnicas state-of-the-art en biología molecular. La identificación de cepas y clones permite elaborar métodos de diagnóstico más sensibles y específicos, puesto que éstos tienen como objetivo los antígenos propios de cada cepa e incluso de cada clon. 
En el área terapéutica, este estudio tendría gran importancia puesto que daría una idea de las cepas circulantes en Centroamérica, datos que pueden ser analizados junto con otros de Latinoamérica y aplicados a la elaboración de una vacuna de ADN preventiva, aunque se requeriría de apoyo internacional para lograr este objetivo. La elaboración de fármacos no tóxicos sería otro aspecto a tener en cuenta, ya que no se cuenta con un tratamiento 100\% efectivo y sin efectos secundarios tan adversos.

\section{Observaciones finales}

La enfermedad de Chagas es un problema primario de salud pública en Nicaragua. Por la naturaleza de la enfermedad, es muy difícil diagnosticar a las personas infectadas. La mayoría de estas personas viven en condiciones de extrema pobreza y el medicamento -que muy pocos reciben- presenta muchos efectos adversos y no les garantiza la cura. Es necesario desarrollar nuevas estrategias de control vectorial, además de invertir en investigación para métodos diagnósticos más sensibles y específicos así como tratamientos más eficaces.

A pesar de que se han aplicado algunos planes de control vectorial y se ha dado educación a algunas comunidades endémicas, la base de datos de pacientes chagásicos está desactualizada. No es posible elaborar métodos de erradicación y control si no se tienen cifras reales de pacientes infectados en el país. No obstante, la falta de recursos es un gran obstáculo para llevar a cabo cualquier intervención.

Se requiere de un esfuerzo conjunto entre el MINSA y los grupos de investigación nicaragüenses para dar solución a este problema, buscando apoyo externo para la aplicación de estrategias y planes de control y erradicación, así como de investigación diagnóstica y terapéutica.

\section{Referencias bibliográficas}

ACEVEDO, F;; GODOY, E. \& SCHOFIELD, C. J. (2000) "Comparison of intervention strategies for control of Triatoma dimidiata in Nicaragua". Memorias do Instituto Oswaldo Cruz 95: 867 - 871.

ANDRADE, S. G.; RASSI, A.; MAGALHAES, J. B.; FERRIOLLI, F. F. \& LUQUETTI, A. O. (1992) "Specific chemotherapy of Chagas' disease: a comparison between the response in patients and experimental animals infected with the same strain". Transactions of the Royal Society of Tropical Medicine and Hygiene 86: $824-826$.

CAMPOS, M. A.; ALMEIDA, I. C.; TAKEUCHI, O.; AKIRA, S.; VALENTE, E. P.; PROCÓPIO, D. O.; TRAVASSOS, L. R.; SMITH, J. A.; GOLENBOCK, D. T. \& GAZZINELLI, R. T. (2001) "Activation of Toll-like receptor 2 by glycosylphosphatidylinositol anchors from a protozoan parasite”. Journal of Immunology 167: 416 - 423.

COURA, J. R. (2007) "Chagas disease: What is known and what is needed - a background article”. Memorias do Instituto Oswaldo Cruz 102 (Supl 1): 113 - 122.

DOYLE, P. S.; ZHOU, Y. M.; ENGEL, J. C. \& MCKERROW, J. H. (2007) "A cysteine protease inhibitor cures Chagas disease in a immunodeficient-mouse model of infection". 
Antimicrobial Agents and Chemotherapy 51: 3932 - 3939.

GADELHA, A. M.; VERCOSA, A. F.; LORENA, V. M.; NAKAZAWA, M.; CARVALHO, A. B.; SOUZA, W.; FERREIRA, A. G.; SILVA, E. D.; KRIEGER, M. A.; GOLDENBER, G. S. \& GOMES, Y. M. (2003) "Chagas' disease diagnosis: comparative analysis of recombinant ELISA with conventional ELISA and hemoaglutination”. Vox Sanguinis 85: 165 - 170.

IDEASS (2004) "Serodiagnostic kit for human leptospirosis and Chagas disease". Iniciative for Development and South-South Cooperation-Niacargua. Disponible en: www. ideassonline.org. Consultado: 6 de octubre de 2008.

JICA (2003) Proyecto Regional de JICA para el control de la enfermedad de Chagas. Folleto informativo del proyecto distribuido por la Agencia de Cooperación Internacional de Japón. Managua: JICA

KIRCHOFF, L. V. (2006) "Chagas' disease" In Harrison: Principles of Internal Medicine. EU: McGraw - Hill International. $16^{\text {ta }}$ edición.

MSF (MÉDICOS SIN FRONTERAS) (2005) Lecciones aprendidas: La enfermedad de Chagas, amenaza invisible en Nicaragua. Folleto informativo de la enfermedad de Chagas. Managua:MSF. $1^{\text {era }}$ edición.

MINSA (2005) "Situación epídemiológica de la enfermedad de Chagas en el SILAIS Madriz, Nicaragua”. Boletín epidemiológico Semana 14. Managua: MINSA.

MORALES, W. G. (2000) "Seroprevalencia de le enfermedad de Chagas en dos comunidades rurales de Nicaragua". Tesis para Master Internacional en Enfermedades Parasitarias Tropicales. Universidad de Valencia, España.

MORRIS,S.A.;TANOWITZ,H.B.;WITTNER,M.\&BILEZIKIAN,J.B.(1990) "Pathophysiological insights into the cardiomyophaty of Chagas disease". Circulation 82: $1900-1909$.

PALMA-GUZMÁN, R.; RIVERA, T. \& MORALES, W. G. (1996) “Domestic vectors of Chagas' disease in three rural communities of Nicaragua". Revista del Instituto de Medicina Tropical de Sao Pablo 38: 133 - 140.

PONCE, C.; PONCE, E.; VINELLI, E.; MONTOYA, A.; DE AGUILAR, V.; GONZÁLEZ, A.; ZINGALES, B.; RANGEL-ALDAO, R.; LEVIN, M. J.; ESFANDIARI, J.; UMEZAWA, E.; LUQUETTI A, D. A. \& SILVEIRA, J.(2005) "Validation of a rapid and reliable test for the diagnosis of Chagas' disease by the detection of Trypanosoma cruzi specific antibodies in blood donors and patients in Central America". Journal of Clinical Microbiology 43: $5065-5068$.

RIVERA, T.; PALMA-GUZMÁN, R. \& MORALES, W. G. (1995) "Seroepidemiological and clinical study of Chagas' disease in Nicaragua”. Revista del Instituto de Medicina Tropical de Sao Pablo 37: 207 - 213.

SHLOMAI, J. (2004) “The structure and replication of kinetoplast DNA". Current Molecular Medicine 4: $623-647$.

STRUM, N.R.et al (1989) "Sensitive detection and schizodeme classification of Trypanosoma cruzi cells by amplification of kinetoplast DNA minicircle sequences: Use in diagnosis of Chagas' disease”. Molecular and Biochemical Parasitology 33: $205-214$.

TAN, H. \& ANDREWS, N. W. (2002) "Don't bother to knock - the cell invasion strategy of Trypanosoma cruzi". Trends in Parasitology 18: 427 - 428.

TARLETON, R. L. (2007) "Immune system recognition of Trypanosoma cruzi". Current Opinion in Immunology 19: 430 - 434.

TYLER, K. M. \& ENGMAN, D. M. (2001) “The life cycle of Trypanosoma cruzi revisited". International Journal of Parasitology 31: 472 - 481. 
URBINA, J. A. \& DOCAMPO, R. (2003) "Specific chemotherapy of Chagas' disease: Controversies and advances". Trends in Parasitology 19: 495 - 501.

VAGO, A. R.; MACEDO, A. M.; OLIVEIRA, R. P.; ANDRADE, L. O.; CHIARI, E.; GALVAO, L.; D’AVILA REIS, D.; PEREIRA, M.; SIMPSON, A.; TOSTES, S. \& PENA, S.(1996) "Kinetoplast DNA signatures of Trypanosoma cruzi strains obtained directly from infected tissues". American Journal of Pathology 146: 2153 - 2159.

VAGO, A. R.; ANDRADE, L. O.; LEITE, A. A.; D’AVILA REIS, D.; MACEDO, A. M.; ADAD, S. J.; TOSTES, S.; MOREIRA, M.; BRASILEIRO FILHO, G. \& PENA, S. (2000) "Genetic characterization of Trypanosoma cruzi directly from tissue of patients with chronic Chagas' disease". American Journal of Pathology 156: 1805 - 1809.

WORLD HEALTH ORGANIZATION (WHO) (2005) Chagas Disease. Disponible en: http:// www.who.int/tdr/sve/diseases/chagas Consultado: 25 de junio de 2008. 